\title{
\begin{tabular}{l|l} 
Mitraries & DSpace@MIT
\end{tabular}
}

\author{
MIT Open Access Articles
}

\section{Clutchable Series-Elastic Actuator: Design of a Robotic Knee Prosthesis for Minimum Energy Consumption}

The MIT Faculty has made this article openly available. Please share how this access benefits you. Your story matters.

Citation: Rouse, Elliott Jay; Mooney, Luke M.; Martinez-Villalpando, Ernesto C.; Herr, Hugh M. "Clutchable Series-Elastic Actuator: Design of a Robotic Knee Prosthesis for Minimum Energy Consumption". 13th International Conference on Rehabilitation Robotics, ICORR 2013.

As Published: http://depts.washington.edu/uwconf/icorr2013/poster_sessions.html

Persistent URL: http://hdl.handle.net/1721.1/81164

Version: Author's final manuscript: final author's manuscript post peer review, without publisher's formatting or copy editing

Terms of use: Creative Commons Attribution-Noncommercial-Share Alike 3.0 


\title{
Clutchable Series-Elastic Actuator: Design of a Robotic Knee Prosthesis for Minimum Energy Consumption
}

\author{
Elliott J. Rouse, Luke M. Mooney, Ernesto C. Martinez-Villalpando and Hugh M. Herr, Members IEEE \\ MIT Media Lab \\ Massachusetts Institute of Technology \\ Cambridge, MA 02139 \\ erouse@media.mit.edu
}

\begin{abstract}
The cyclic and often linear torque-angle relationship of locomotion presents the opportunity to innovate on the design of traditional series-elastic actuators (SEAs). In this paper, a novel modification to the SEA architecture was proposed by adding a clutch in parallel with the motor within the SEA-denoted as a CSEA. This addition permits bimodal dynamics where the system is characterized by an SEA when the clutch is disengaged and a passive spring when the clutch is engaged. The purpose of the parallel clutch was to provide the ability to store energy in a tuned series spring, while requiring only reactionary torque from the clutch. Thus, when the clutch is engaged, a tuned elastic relationship can be achieved with minimal electrical energy consumption. The state-based model of the CSEA is introduced and the implementation of the CSEA mechanism in a powered knee prosthesis is detailed. The series elasticity was optimized to fit the spring-like torqueangle relationship of early stance phase knee flexion and extension during level ground walking. In simulation, the CSEA knee required $70 \%$ less electrical energy than a traditional SEA. Future work will focus on the mechanical implementation of the CSEA knee and an empirical demonstration of reduced electrical energy consumption during walking.
\end{abstract}

Keywords-series elastic actuator, clutch, lower limb prosthesis, powered prosthesis, biologically inspired design

\section{INTRODUCTION}

Over the past two decades, series-elastic actuators (SEAs) have been the focus of much investigation in the development of force controlled robots [1-6]. SEAs include the addition of a series compliance between the transmission output and load, and have been shown to have many advantages. These advantages include increased shock tolerance, energy storage and limited high-frequency actuator impedance, among others [1, 2, 7]. Furthermore, the series compliance increases force fidelity, a property especially useful in the impedance controlled applications in fields such as wearable robotics and human-machine interfacing [8-10].

SEAs have been implemented successfully in lower extremity prosthetic and exoskeleton devices. Au et al. used

This work was supported by Department of Defense award number W81XWH-09-2-0143 and by the National Science Foundation Graduate Research Fellowship award number 1122374. series and parallel elasticity in the design of a powered ankle prosthesis [10], which was shown to lower the metabolic cost of walking in transtibial amputees [11]. Additionally, Veneman et al. [9] designed a lower extremity exoskeleton using Bowden cable driven SEAs. Recently, a new iteration of the design has been proposed [12] using a direct-mounted SEA rather than a Bowden cable drive. These devices capitalize on the benefits of SEAs, but the cyclic and often spring-like torque-angle relationship observed during locomotion presents an opportunity to further innovate the SEA architecture.

One example of a cyclic, spring-like joint torque behavior in legged locomotion occurs during early-stance, knee flexion/extension phases of human locomotion [13]. Here, an SEA implemented with a series stiffness approximately equal to the slope of the torque-angle relationship during this phase (often denoted as the quasistiffness [14]) would provide a reduction in the work required by the electric motor within the SEA. In other words, the complete torque-angle relationship would be rendered by the series stiffness alone, requiring only reactionary torque to be generated by the motor, at negligible velocity. This provides a decrease in the electrical energy required by the motor; however, because electric motors are not efficient at low velocities, further improvements can be made. To address this issue, Haeufle et al. [15] recently introduced a clutched parallel-elastic actuator. In their work, a spring was incorporated in parallel to the electric motor and was engaged with a clutch. The purpose of the parallel spring was to augment the torque-angle characteristics of the mechanism passively. Such a design is advantageous because it enables a reduced gear ratio and less powerful motor to be used, while still maintaining the proper kinematics and kinetics of stance phase. However, because the motor was directly coupled to the output, it cannot take advantage of the aforementioned beneficial properties of an SEA. Furthermore, because human locomotion includes spontaneous high-power modes (e.g. ascending stairs and sitto-stand transitions), it is essential for a prosthesis to be able to provide significant output torque and velocity. Thus, the potential clinical benefit provided by the parallel spring design is likely outweighed by its inability to provide a complete array of locomotory modes required during activities of daily living. 

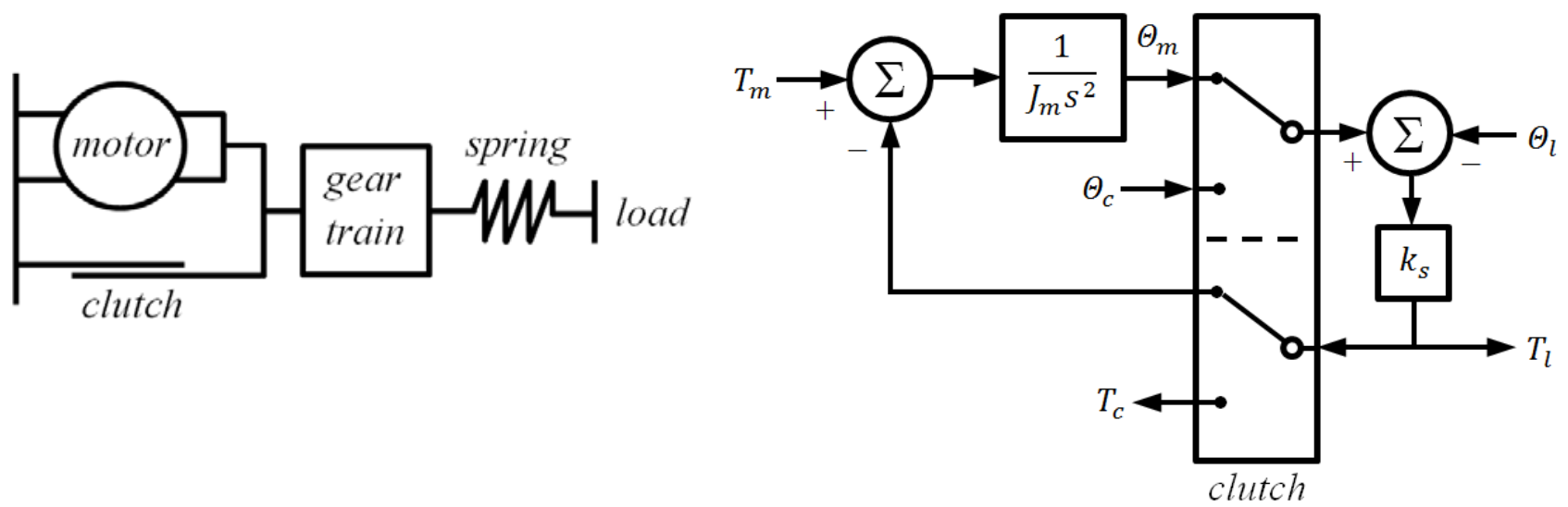

Fig. 1. Left: Model of Clutchable Series-Elastic Actuator shown. The clutch is in parallel with the motor such that when it is engaged, no load is required from the motor. Right: Frequency domain diagram of the CSEA (modified from [2]) shown with switches engaged by the activation of the clutch, where $\Theta$ denotes position, $T$ denotes torque, subscripts $m, l$ and $c$ denote motor, load and clutch, respectively; finally, $k_{s}$ is the series spring stiffness and $J_{m}$ is the motor inertia.

In this paper we introduce a new mechanism known as a Clutchable Series-Elastic Actuator (CSEA). The purpose of this device is to take advantage of both the properties of an SEA, as well provide the ability to store energy in a tuned series spring, when the desired task dynamics are elastically conservative in the torque-angle domain. The clutch was added to permit low electrical power consumption while providing the reactionary torque needed. The architecture of this mechanism was motivated by the previous work of Herr et al. [16] and Endo et al. [17]. In the work of [17], agreement was shown between the kinetics and kinematics of a quasi-passive, spring-clutch walking model and biomechanical data for humans ambulating across a level ground surface, underscoring the importance of series-elastic clutch behaviors in human walking. Finally, in this paper, the CSEA concept was implemented in the design of a robotic knee prosthesis. Computationally, the design specifications of the CSEA knee prosthesis are detailed, providing insight into one possible implementation of the CSEA architecture in wearable robotics.

\section{Mechansim Design}

The CSEA mechanism was motived by the ability to provide the benefits of an SEA, while simultaneously, providing low-energy, elastic behaviors when a mechanical clutch is engaged. Furthermore, the series stiffness should be tuned to provide a specific torque-angle (or forcedisplacement) relationship observed prior to its implementation in an application. During this period of a conservative torque-angle relationship, the clutch is activated to provide the reaction torque. As a result of the tuned series elasticity, the output kinematics will follow the specified elastic dynamics. The CSEA mechanism consists of such a tuned series compliance within a standard SEA with an added clutch in parallel with the motor. A model of the CSEA design is shown in Fig. 1 (left) and the frequency domain diagram is shown on the right. From this diagram we can obtain the following model in the time domain:

$$
\begin{aligned}
\tau_{m} & = \begin{cases}J_{m} \ddot{\theta}_{m}+\frac{\tau_{1}}{N \eta} & \text { clutch off } \\
0 & \text { clutch on }\end{cases} \\
\theta_{m} & = \begin{cases}\frac{\tau_{l}}{k_{s}}+\theta_{l} & \text { clutch off } \\
\theta_{c} & \text { clutch on }\end{cases}
\end{aligned}
$$

where $\tau_{l}$ is the torque on the load, $\tau_{m}$ is the torque on the motor, $k_{s}$ is the series spring stiffness, $\theta_{l}, \theta_{m}$ and $\theta_{c}$ are the positions of the load, motor and clutch, respectively; $N$ is the gear ratio, $\eta$ is the gear train efficiency and, finally, $J_{m}$ is the motor's rotor inertia. When the clutch is activated, the equations governing the dynamics change, and the mechanism behaves with passive-elastic dynamics.

Using the electrical model analogy for a motor, the equations governing the electrical power consumption can be obtained from (1) and (2):

$$
\begin{aligned}
& i_{m}= \begin{cases}\frac{\tau_{m}}{k_{t}} & \text { clutch off } \\
i_{c} & \text { clutch on }\end{cases} \\
& v_{m}= \begin{cases}\frac{R_{m} J_{m}}{k_{t}} \ddot{\theta}_{m}+\frac{\dot{\theta}_{m}}{k_{v}}+\frac{R_{m}}{k_{t}} \tau_{m} & \text { clutch off } \\
v_{c} & \text { clutch on }\end{cases}
\end{aligned}
$$

where $k_{t}$ is the torque constant of the motor, $R_{m}$ is the winding resistance and $k_{v}$ is the speed constant of the motor. The current and voltage required to activate the clutch is $i_{c}$ and $v_{c}$, respectively. The energetic economy comes from the difference in the electrical power required by the motor and the clutch, integrated over the period of activation. As a 

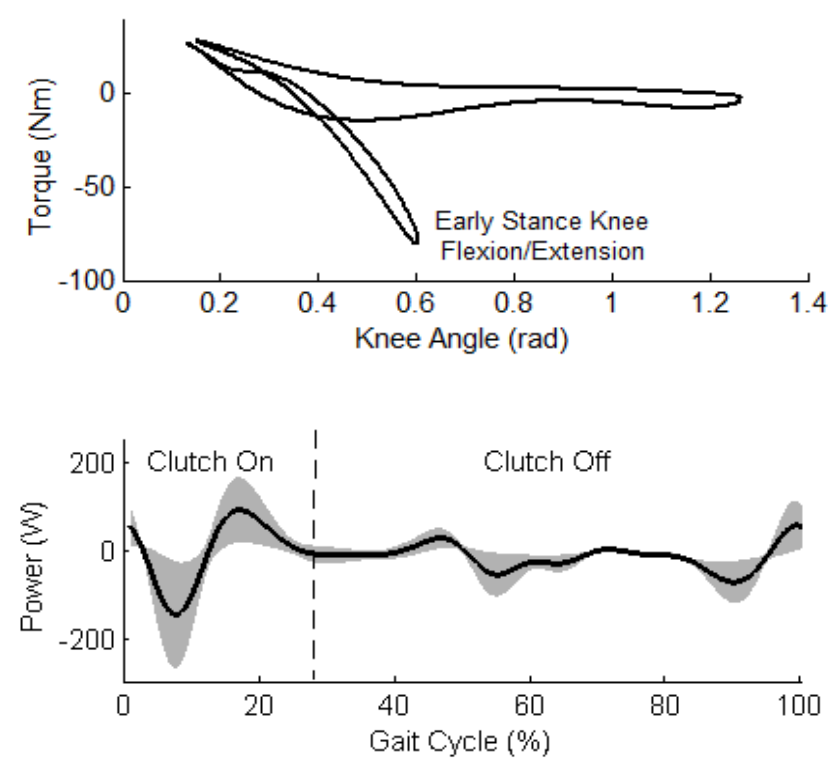

Fig. 2. A) Knee torque as a function of angle shown for a single stride of a representative human subject. Note the linear, near zero net work region of early stance phase knee flexion/extension. B) Knee power during the gait cycle shown averaged across subjects and walking speeds. Bold denotes intersubject average and trannslucent is one standard deviation. The 'clutch on' portion shown is the early stance knee flexion/extension portion of the gait cycle and the clutch would remain off throughout the rest of the gait cycle. From these plots, the potential energetic economy of the proposed CSEA design can be understood.

result of the clutch being placed before the gear train, a low power clutch may be used. For example, electromagnetic or spring-wrap clutches operate at very low power consumption (less than six watts) for holding torques between one and three newton-meters. Therefore, the potential energetic advantage of the CSEA can be substantial.

\section{IMPLEMENTATION IN A PROSTHETIC KNEE}

To demonstrate the potential of the CSEA to reduce electrical energy requirements, it was implemented in the theoretical design of a robotic prosthetic knee. A knee prosthesis was chosen because there is a portion of stance phase of walking that is able to take advantage of the CSEA architecture. That is, during early stance phase knee flexion and extension, the knee torque-angle relationship is predominantly spring-like in character (Fig. 2A). Additionally, this portion of stance phase is linear and consists of the greatest positive (and negative) mechanical power phases required during the gait cycle, further demonstrating the potential utility of the CSEA in the design of a robotic knee prosthesis (Fig. 2B).

\section{A. Pilot Data Collection for Simulations}

For use in estimating the kinetics and kinematics necessary during the gait cycle, pilot data were collected at
TABle I: CSEA Model PARAMETERS

\begin{tabular}{ccc}
\hline \hline Parameter & Symbol & Value \\
\hline Motor Inertia & $J_{m}$ & $33.3 \mathrm{gcm}^{2}$ \\
Gear Ratio & $N$ & 145 \\
Mechanical Efficiency & $\eta$ & 0.9 \\
Torque Constant & $k_{t}$ & $20.6 \mathrm{mNm} / \mathrm{A}$ \\
Speed Constant & $k_{v}$ & $48.8 \mathrm{rad} / \mathrm{s} / \mathrm{V}$ \\
Winding Resistance & $R_{m}$ & $0.21 \Omega$ \\
Clutch Current & $i_{b}$ & $0.25 \mathrm{~A}$ \\
Clutch Voltage & $v_{b}$ & $24 \mathrm{~V}$ \\
Series Stiffness & $k_{s}$ & $218 \mathrm{Nm} / \mathrm{rad}$ \\
\hline \hline
\end{tabular}

the Harvard University Skeletal Biology Lab in a study approved by the MIT Committee On the Use of Humans as Experimental Subjects. Acquired data were used to provide information for subjects across a range of speeds and weights. After obtaining informed consent, three participants were asked to walk barefoot on an instrumented treadmill for two minutes at $0.75 \mathrm{~m} / \mathrm{s}, 1.5 \mathrm{~m} / \mathrm{s}$ and $2.0 \mathrm{~m} / \mathrm{s}$. The kinetic data were collected using the force plates of the treadmill (Bertec Corporation, Columbus OH) and sampled at $1000 \mathrm{~Hz}$ while the kinematic data were collected synchronously via an infrared camera system (eight cameras, Qualisys Motion Capture Systems, Gothenburg, Sweden) and sampled at 500 Hz. The motion capture system utilized passive markers placed at 42 (bilateral) locations on the participant's body according to the Helen Hayes marker model. The raw data

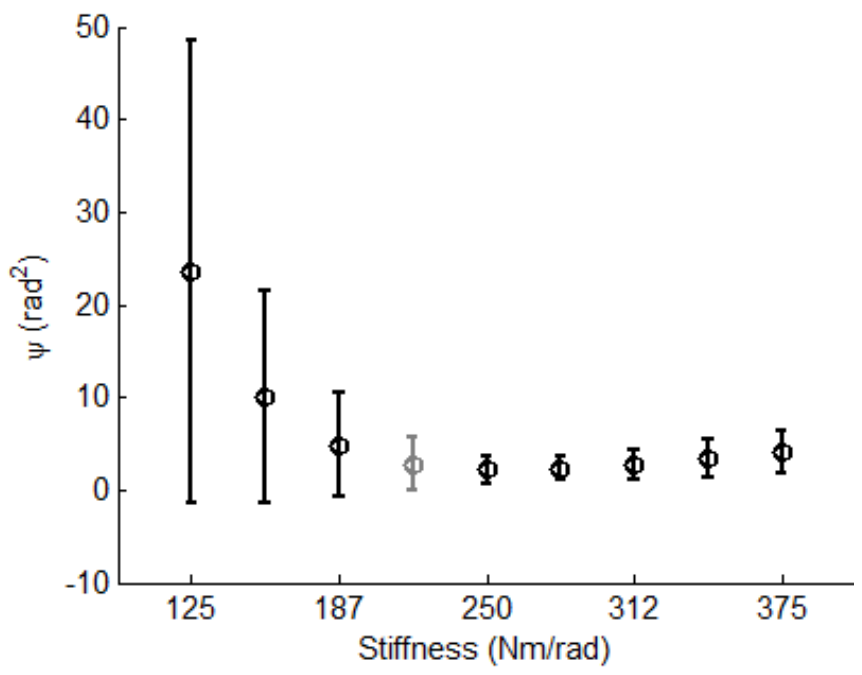

Fig. 3. Model agreement shown as a function of series stiffness, averaged across subjects and walking speeds. Error bars denote standard deviation. The stiffness implemented in the CSEA knee prosthesis is shown in gray and listed in Table 1. 
were processed in SIMM (Musculographics Inc., Evanston, IL) to obtain joint trajectories and dynamics.

\section{B. Stiffness of Series Elasticity}

To estimate the optimal stiffness, the aforementioned model of the CSEA was used (see Mechanism Design subsection and Table 1). The pilot data were kinetically clamped and the clutch was activated during early stance phase knee flexion/extension. It was specified to become activated and deactivated at the closest point of zero velocity. Switching at zero velocity minimized the effect of the discontinuous dynamics. It should be noted that in this analysis, each configuration of stiffness yields essentially identical electrical power profiles, but very different knee kinematics; hence the focus of comparison will be on kinematic similarity rather than electrical energy.

As a result of the varying torque-angle relationships across subjects and walking speeds, the kinematic effect was assessed. The agreement between the CSEA model and the experimental data were determined as a function of the series stiffness, where angular stiffness is referenced to the output. In other words, the kinematic effect of varying series stiffness was assessed, with the optimal stiffness closely matching knee kinematics. The scalar kinematic agreement, $\psi$, was defined by

$\psi=\int\left(\theta_{C S E A}-\theta_{l}\right)^{2} d t$

where $\theta_{C S E A}$ is the output displacement of the CSEA and the load displacement, $\theta_{l}$, is the knee angle; lastly, the function was integrated over the length of each gait cycle. The agreement was determined for each subject and walking speed (Fig. 3).

The softer series stiffness caused substantial knee flexion values beyond the profiles acquired during level ground walking, whereas the greater stiffness values tracked the kinematics more closely, but reduce the benefits of including the series elasticity. Thus, in addition to practical design factors, the stiffness implemented in the CSEA knee was chosen to be $218 \mathrm{Nm} / \mathrm{rad}$, shown in gray.

\section{Energy Efficiency}

Using the optimal series stiffness, the CSEA model was shown to be significantly more energy efficient during the gait cycle. The pilot data were kinetically clamped and the motor torque and position were estimated during stance phase from the equations (1) and (2). Using equations (3) and (4), the electrical power required throughout stance phase was determined (Fig. 4). The clutch was activated at the closest points of zero velocity during early stance phase knee flexion. The required electrical energy was determined for each subject for traditional SEA and CSEA designs, with no electrical power regeneration. A two sample t-test was used to show the difference in energy, with a 0.05 level of significance.

The CSEA design outperformed the traditional SEA design, requiring less energy, when averaged across subjects and walking speeds (Fig. 5). The CSEA on average required $70 \%$ less energy per stride and was statistically less than the traditional SEA design $(p=0.024)$. The large standard deviation denotes the substantial variance in power required for subjects of varying bodyweight to walk at differing speeds.
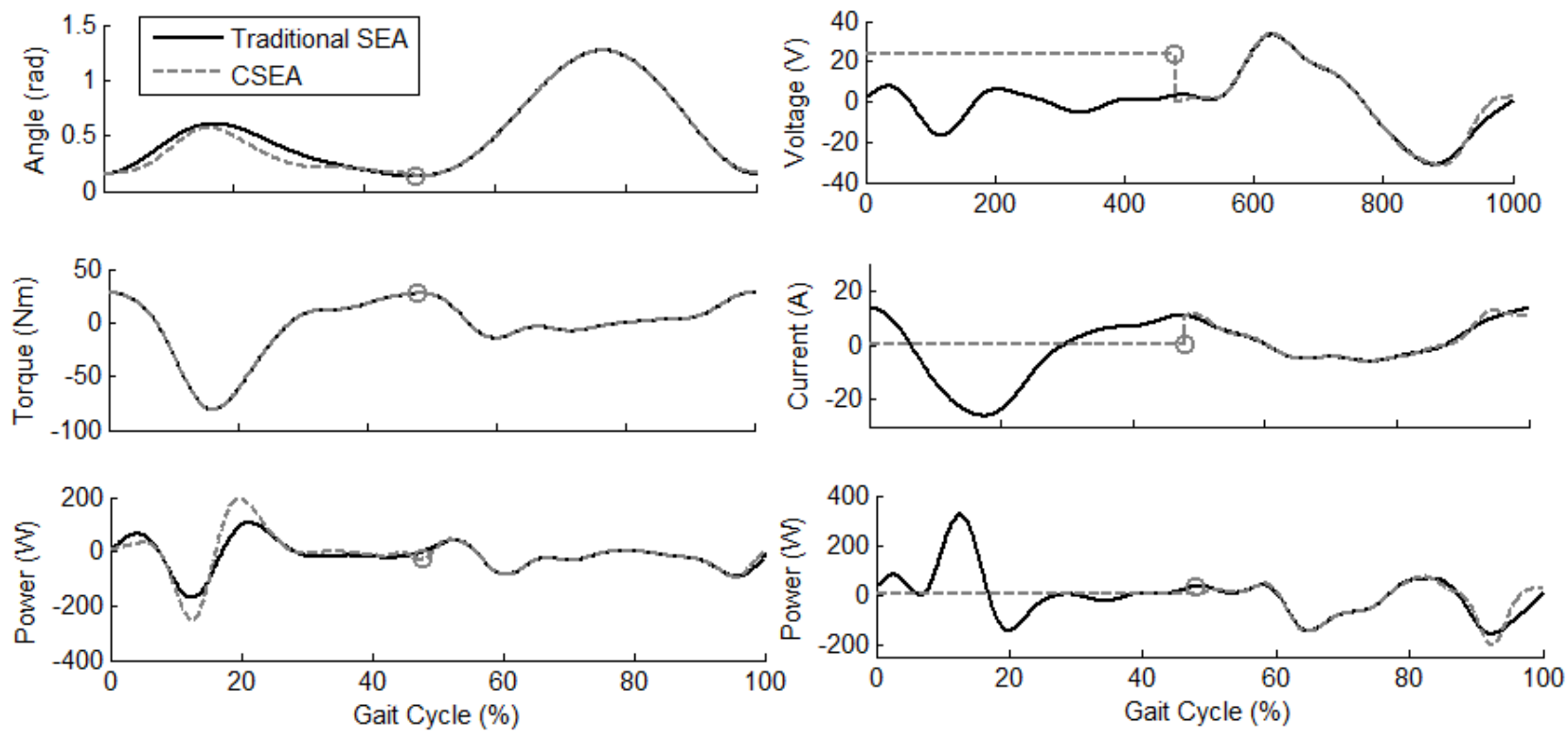

Fig. 4. Left: Knee kinetic and kinematic data shown for a representative subject at $1.5 \mathrm{~m} / \mathrm{s}$ for both the CSEA and a traditional SEA implementations. The differing values can be seen during the period of clutch activation (prior to the gray circle), and includes a slight reduction in the ideal magnitude of the early stance knee flexion angle. Right: The electrical power required to produce the kinetic and kinematic data during walking using both the CSEA and a traditional SEA. Note the static values of power variables during the period of clutch activation, with mechanical power shown on the left and electrical power shown on the right. 


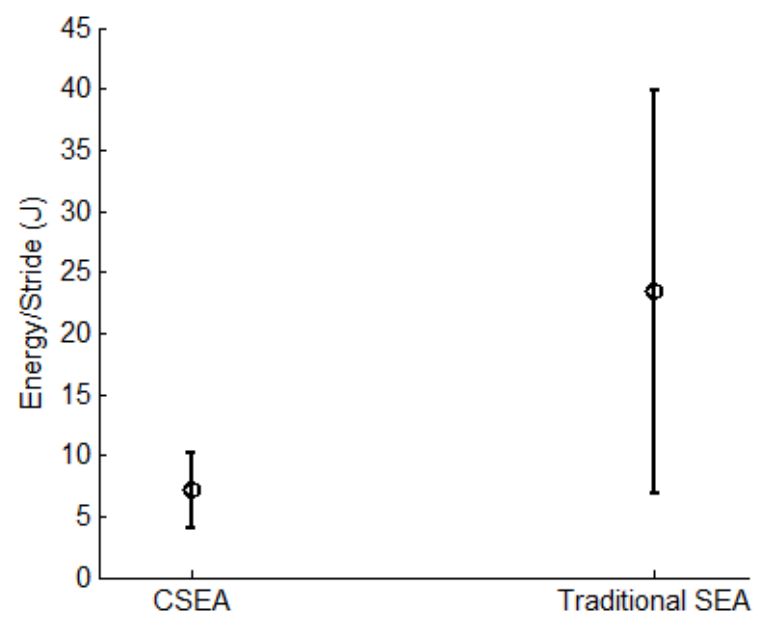

Fig. 5. Intersubject average electrical energy consumed during a single stride, with errorbars denoting standard deviation across subjects and walking speeds. The CSEA required consistently less energy across subjects and walking speeds.

\section{DISCUSSION}

In this paper, a novel modification of the SEA was introduced, known as the Clutchable Series-Elastic Actuator. In this design, a clutch was placed in parallel with the traditional SEA motor, permitting bimodal dynamics - the CSEA is able to behave as an SEA when the clutch is disengaged and as a spring when the clutch is engaged. The model structure and equations governing the implementation were provided, in addition to the details of a CSEA used in the design of a robotic knee prosthesis. The CSEA knee was shown to significantly reduce the energy required for level ground walking by $70 \%$.

The CSEA was particularly well suited to take advantage of the linear region of the knee torque-angle relationship in human locomotion, often observed in wearable robotic applications. The series elasticity was chosen to mimic this linear region in stance phase knee flexion/extension, and the clutch was engaged during this period to promote electrical efficiency. It should be noted that there is a second semilinear region of the knee's torque angle relationship (i.e. swing phase knee flexion/extension). However, due to the limited torque needed during this region, it is not necessary to consider this region when tuning the series spring.

Since the electrical efficiency is a result of the addition of the clutch, the motor can be sized to the standard power requirements. During locomotion, there are spontaneous high-power modes that are required and the current design permits the use of a motor capable of these tasks. This is in contrast to the work of Haeufle et al. [15], where a low power motor would be used to take advantage of the parallel spring added in their design. Thus, the CSEA is able to provide a wide spectrum of locomotory modes, despite being tuned for level ground walking.

The series stiffness implemented in the CSEA will affect the magnitude of early stance phase flexion. Increased walking speed has been shown to increase the torque required by the knee [18]. This, combined with greater bodyweight, will increase the peak angle of knee flexion in the CSEA knee due to series spring compression. The CSEA mechanism can easily capture and return this energy; thus, as the walking speed increases, the CSEA knee becomes more efficient when compared to a traditional SEA. This is, however, a tradeoff as the knee flexion angle can be substantially greater than able-bodied kinematics. A possible solution would be to modify the linear tuned series stiffness assumed in this study with a nonlinear hardening stiffness where series stiffness increases with increasing spring deflection.

One limitation of the CSEA mechanism was the increased reflected inertia from the added clutch and the increased design complexity. By including the clutch before the gear train, it is able to hold a large output torque with a relatively small, low power clutch. This benefit comes at the cost of increasing the reflected inertia, as the clutch is in parallel with the motor. Increased reflected inertia decreases energetic efficiency. Thus, the implementation of the clutch and tradeoff with reflected inertia and design complexity must be considered carefully for each application.

Future work will consist of the hardware development of the CSEA knee. The design theory of the knee indicates it will have substantial energetic advantage over current technologies. This will permit the use of a smaller battery, thereby reducing the overall prostheses weight - a critical issue in the development of robotic prosthetic applications.

\section{REFERENCES}

[1] Pratt, G.A., and Williamson, M.M.: 'Series elastic actuators', Intelligent Robots and Systems 95.'Human Robot Interaction and Cooperative Robots', Proceedings. 1995 IEEE/RSJ International Conference on, 1995, 1, pp. 399-406

[2] Pratt, G., Williamson, M., Dillworth, P., Pratt, J., and Wright, A. 'Stiffness isn't everything', Experimental Robotics IV, 1997, pp. 253262

[3] Robinson, D.W., Pratt, J.E., Paluska, D.J., and Pratt, G.A.: 'Series elastic actuator development for a biomimetic walking robot', Advanced Intelligent Mechatronics, 1999. Proceedings. 1999 IEEE/ASME International Conference on, 1999, pp. 561-568

[4] Pratt, J., Krupp, B., and Morse, C.: 'Series elastic actuators for high fidelity force control', Industrial Robot: An International Journal, 2002, 29, (3), pp. 234-241

[5] Sensinger, J.W., and Weir, R.F.: 'Design and analysis of a nonbackdrivable series elastic actuator', Rehabilitation Robotics, 2005. ICORR 2005. 9th International Conference on, 2005, pp. 390-393

[6] Albu-Schaffer, A., Eiberger, O., Grebenstein, M., Haddadin, S., Ott, C., Wimbock, T., Wolf, S., and Hirzinger, G.: 'Soft robotics', Robotics \& Automation Magazine, IEEE, 2008, 15, (3), pp. 20-30

[7] Sensinger, J.W.: 'Improvements to series elastic actuators', Mechatronic and Embedded Systems and Applications, Proceedings of the 2nd IEEE/ASME International Conference on, 2006, pp. 1-7

[8] Sensinger, J.W., and Weir, R.F.f.: 'User-Modulated Impedance Control of a Prosthetic Elbow in Unconstrained, Perturbed Motion', Biomedical Engineering, IEEE Transactions on, 2008, 55, (3), pp. 1043-1055

[9] Veneman, J., Ekkelenkamp, R., Kruidhof, R., Van Der Helm, F., and Van Der Kooij, H.: 'A series elastic-and bowden-cable-based actuation system for use as torque actuator in exoskeleton-type robots', The International journal of robotics research, 2006, 25, (3), pp. $261-281$ 
[10] Au, S., and Herr, H.: 'On the design of a powered ankle-foot prosthesis. The Importance of series and parallel motor elasticity', IEEE Robotics and Automation Magazine, 2008, 15, (3), pp. 52-59

[11] Au, S.K., Weber, J., and Herr, H.: 'Powered Ankle--Foot Prosthesis Improves Walking Metabolic Economy', Robotics, IEEE Transactions on, 2009, 25, (1), pp. 51-66

[12] Lagoda, C., Schouten, A.C., Stienen, A.H.A., Hekman, E.E.G., and van der Kooij, H.: 'Design of an electric series elastic actuated joint for robotic gait rehabilitation training', Biomedical Robotics and Biomechatronics (BioRob), IEEE RAS and EMBS International Conference on, 2010, pp. 21-26

[13] Winter, D.A.: 'Biomechanics and motor control of human movement' (Wiley, 2009. 2009)

[14] Rouse, E.J., Gregg, R.D., Hargrove, L.J., and Sensinger, J.W.: 'The difference between stiffness and quasi-stiffness in the context of biomechanical modeling', IEEE Trans. Biomed. Eng., 2012, 60, (2), pp. 562-568
[15] Haeufle, D., Taylor, M., Schmitt, S., and Geyer, H.: 'A clutched parallel elastic actuator concept: towards energy efficient powered legs in prosthetics and robotics', Biomedical Robotics and Biomechatronics (BioRob), IEEE RAS \& EMBS International Conference on, 2012, pp. 1614-1619

[16] Herr, H.M., Paluska, D.J., and Dilworth, P.: 'Artificial human limbs and joints employing actuators, springs, and variable-damper elements'2006

[17] Endo, K., Paluska, D., and Herr, H.: 'A quasi-passive model of human leg function in level-ground walking', Intelligent Robots and Systems, IEEE/RSJ International Conference on, 2006, pp. 49354939

[18] Kirtley, C., Whittle, M.W., and Jefferson, R.: 'Influence of walking speed on gait parameters', J. Biomed. Eng., 1985, 7, (4), pp. 282-288 